\title{
Notas críticas sobre el discurso del postdesarrollo
}

\section{Introducción}

Al comienzo de un nuevo siglo, existe una profunda sensación de que nos encontramos al final de una era. La manifestación más extrema de este pensamiento apocalíptico se puede encontrar en los movimientos religiosos de carácter fundamentalista diseminados alrededor del mundo. En el mundo académico, lo anterior se expresa en una serie de enfoques que estudian lo social y lo cultural, los cuales tienen sus propias versiones de las viejas finalizaciones y los nuevos comienzos, y los términos "post" y "nuevo" son de los términos más utilizados en su retórica. Este artículo examina un ejemplo concreto de esta forma de percibir el presente: el discurso del postdesarrollo (Rahnema y Bawtree, 1997). Varios autores han identificado un impasse en la teoría del desarrollo (Booth, 1994; Kiely, 1995), vinculando esta situación a la creciente diversidad del llamado Tercer Mundo, al colapso del "socialismo real", a la percepción de una crisis en el análisis marxista y al fracaso de la teoría radical del desarrollo (llámese marxismo o teoría de la dependencia) para ofrecer una alternativa constructiva a la ortodoxia neoliberal' .

Mientras la mayoría de estos autores ${ }^{2}$ hacen un llamado para la estructuración de nuevas teorías del desarrollo que sean más sensibles a los aspectos de agencia y diversidad en las sociedades periféricas, la teoría del postdesarrollo ofrece ostensiblemente una alternativa más radical a la teoría del desarrollo - un rompimiento completo con la idea del desarrollo (Sachs, 1992; Escobar, 1995). Influenciada por varias corrientes del postestructuralismo, feminismo ambientalista, teoría postcolonial y (como ya veremos) populismo romántico, esta teoría afirma que la idea del desarrollo constituye una manera específica de pensar acerca de la

* Conferencista del Departamento de Sociología y Estudios Sociales de la Universidad de Regina (Canadá) y Profesor de la Macstría cn Gestión del Medio Ambicnte de la Universidad Centroamericana "José Simeón Cañas" (UCA). 
realidad, una forma particular de conocimiento. Desarrollo es, siguiendo las ideas de Foucault, un discurso particular que no sólo refleja, sino realmente construye una realidad. Al hacerlo, cierra la posibilidad de cualquier pensamiento alternativo, y por lo tanto constituye una forma de poder. De esta forma, "el Tercer Mundo... [es] producido por los discursos y las prácticas del desarrollo desde su incorporación en el período inmediato a la finalización de la Segunda Guerra Mundial" (Escobar 1995: 4).

Este artículo ofrece una evaluación sociológica-crítica del discurso del postdesarrollo. En primer lugar, se comienza por delinear en detalle los intentos recientes de deconstruir la idea del desarrollo y la propuesta de la novedosa noción acerca del futuro del "postdesarrollo". A pesar de simpatizar con los elementos de la crítica que este enfoque desarrolla — su énfasis en la contingencia y la neutralidad de la tecnocracia- nuestro argumento sugiere que la noción de postdesarrollo es en sí misma imperfecta. La segunda sección examina los problemas con la noción del postdesarrollo, enfocándose en los problemas metodológicos, el problema de la agencia y de su política. Finalmente, se concluye indicando las bases para un enfoque alternativo al discurso del desarrollo.

\section{La noción del postdesarrollo}

Si bien se puede que sus vestigios pueden encontrarse en el siglo XIX, fue en el período posterior a la Segunda Guerra Mundial que la noción del desarrollo fue elaborada explícitamente. En 1949, el presidente estadounidense Truman, en su discurso inaugural, señaló que existía la necesidad que los países del mundo moderno adoptaran un alto grado de responsabilidad en resolver los problemas de las regiones subdesarrolladas.

Más de la mitad de la población mundial está viviendo en condiciones cercanas a la miseria. Su alimentación es inadecuada, son víctimas de las enfermedades. Su vida económica es primitiva y estancada. Su pobreza es una desventaja y una amenaza tanto para ellos como para las áreas más prósperas. Por primera vez en la historia la humanidad posee el conocimiento y la destreza para aliviar los sufrimientos de estos pueblos... Creo que debemos poner a disposición a esta gente amante de la paz los beneficios de nuestro inventario de conocimiento técnico para ayudarlos a realizar sus aspiraciones para una vida mejor.

... Lo que nosotros prevemos es un programa de desarrollo basado en los conceptos del trato justo y democrático... Una mayor producción es la clave para la prosperidad y la paz. Y la clave para una mayor producción es una amplia y vigorosa aplicación del conocimiento científico y tecnológico moderno (citado por Escobar, 1995: 3). 
Esta famosa alocución de Truman es una clara afirmación del pensamiento básico detrás de la noción de desarrollo?. El Tercer Mundo fue caracterizado como atrasado y primitivo, pero estos problemas — se declaraba - podrían ser superados siguiendo un camino similar de desarrollo que fuera adoptado por los países "civilizados" de Occidente. En efecto, este camino podría recorrerse más fácilmente porque el Occidente podría compartir los beneficios de su prosperidad material y conocimiento científico con las regiones "atrasadas" y de este modo apresurar su transición a la modemidad. Sobre todo, esto podría lograrse a través de un incremento en la producción en las regiones subdesarrolladas, y esto, eventualmente podría ocurrir a través de la introducción de métodos científicos y racionales bajo la tutela de las naciones avanzadas.

Desde 1945, diferentes estrategias han sido empleadas para promover el desarrollo. Para decirlo brevemente, el período comprendido entre fines de la década de los cuarenta a los sesenta del siglo pasado, fue dominado por los enfoques desarrollistas en los que el Estado jugaba un papel protagónico en la promoción de la industrialización (Prebish, 1959). En los setenta se produjeron algunos intentos limitados de promover el desarrollo de acuerdo a las necesidades básicas, lo cual se suponía sería una estrategia de redistribución con crecimiento (Chenery et al., 1974). A fines de los ochenta emergió la contraofensiva neoliberal en la que a los Estados se les estimuló a minimizar su intervención en la esfera económica, y el desarrollo, se afirmaba, iba a lograrse por medio del logro de las ventajas comparativas en la economía mundial en el contexto de una supuesta competencia abierta e irrestricta (Lal, 1984).

Sin embargo, los autores del postdesarrollo argumentan que las disputas sobre las diferentes estrategias de desarrollo resultan menos importantes que los presupuestos compartidos que unifican estos enfoques. Según su punto de vista, cada uno de éstos asume que el desarrollo —en alguna forma- es la solución, mientras que la tendencia del postdesarrollo argumenta que dicha meta es parte del problema. Las diferencias en política, se dice, son "demasiado débiles para cuestionar la ideología del desarrollo y su relevancia para las aspiraciones de la gente" (Rahnema 1997: ix).

Claramente influenciado por el postestructuralismo, Escobar (1995:9) argumenta que el discurso del desarrollo ha "desplegado un régimen de gobierno sobre el Tercer Mundo, un "espacio para seres-sujetos" que asegura el control sobre ellos. Esta concepción de gobernabilidad es muy cercana a la concepción que Foucault tiene de poder y discurso. Para Foucault, un discurso "permite" ciertas formas de pensamiento acerca de algo, y de ese modo excluye otras. En tal sentido, es el discurso, y no el sujeto individual, el que produce el conocimiento —en verdad, el sujeto es producto del discurso (Foucault 1980: 115)-. El conocimiento es, por lo tanto, inseparable del poder - no hay una relación de poder sin la correlativa construcción de un campo de conocimiento, ni cualquier conoci- 
miento que no presuponga y constituya al mismo tiempo, relaciones de poder (Foucault 1977:27). Todas las formas de conocimiento están íntimamente conectadas a relaciones de poder y por lo tanto lo que es verdad no está fuera del poder... Cada sociedad tiene su régimen de verdad, su "política general" de lo que es verdad; esto es, los tipos de discurso que son aceptados y que cumplen la función de expresar lo verdadero" (Foucault 1980: 131).

Así, Escobar es sumamente perspicaz al distinguir entre desarrollo como discurso y desarrollo como ideología -en el sentido marxista—. En tal sentido afirma:

El discurso del desarrollo no es meramente una "ideología" que tiene poco que ver con el "mundo real"; tampoco es un aparato producido por los que ostentan el poder para ocultar otra verdad más básica, llámesele la cruda realidad del signo del dólar. El discurso del desarrollo se ha cristalizado en prácticas que contribuyen a regular las idas y venidas cotidianas de la gente en el Tercer Mundo (Escobar 1995: 104).

La idea del desarrollo, por lo tanto, se cristaliza en un discurso, en una forma particular de pensar acerca de la realidad que excluye cualquier alternativa. Está íntimamente conectado a las relaciones de poder, y en particular, es un medio a través del cual el Occidente continua ejerciendo su dominio en el sistema mundial. El desarrollo, efectivamente, construye una realidad en la que, incluso supuestas concepciones altemativas, se encuadran dentro del mismo discurso. Define "problemas", tales como pobreza, baja productividad en la agricultura y crecimiento poblacional, los cuales solamente pueden ser solucionados a través del progreso. El efecto del discurso del desarrollo de esta forma es la "creación de un espacio de pensamiento y acción, la expansión de lo cual fue dictado con anticipación (Escobar 1995: 42). Así, por ejemplo, una vez que la pobreza ha sido construida como problema, su alivio demanda la intervención del gobierno y de las agencias de ayuda, como consecuencia lógica la "industria del desarrollo" es creada. Al respecto, Escobar asegura que

La pobreza a escala global fue un descubrimiento del período posterior a la II Guerra Mundial ... Si dentro de las sociedades de mercado el pobre fue definido como al que le hacía falta lo que el rico tenía en términos de dinero y posesiones materiales, los países pobres llegaron a ser definidos igualmente en relación a los estándares de riqueza de las naciones económicamente más avanzadas (Escobar 1995: 22-23).

Para los autores del postdesarrollo, entonces, la noción del desarrollo es eurocéntrica, una forma de imperialismo cultural (Sachs, 1992). El Tercer Mundo es identificado como una construcción cultural del Occidente, en algún sentido identificado como sociedades a las que falta lo que el Occidente desarrollado tiene. La diferencia es identificada con atraso, de modo que "las virtudes de la simplicidad y la convivencia, de formas nobles de propiedad, de sabiduría, de apoyarse uno en otro... son ridiculizados como signos de 'subdesarrollo"' 
(Rahnema 1997: x). Escobar, rechazando el punto de vista de Habermas, argumenta que "en el Tercer Mundo, la modernidad no es "un proyecto inacabado del Iluminismo", sino que el desarrollo es el último y fracasado intento del Iluminismo en Asia, África y América Latina" (Escobar 1995: 221).

A diferencia de Escobar, otros autores del postdesarrollo han intentado ligar este discurso a los intereses económicos de las potencias occidentales. Si bien no se ha formulado claramente, pareciera que, desde esta perspectiva, el desarrollo es una ideología (más que un discurso) diseñada para servir los intereses atrincherados y/o reforzar la dominación de Occidente en el sistema mundial, o sea una forma de "intervencionismo arrogante" (Sachs 1992: 2). En la misma línea de argumentación, Rahnema (1997: ix) sostiene que "como en el caso de los examos coloniales, éstas (las potencias de Occidente) se encuentran en la búsqueda de un nuevo sistema de dominación, con la esperanza de que les permitirá mantener su presencia en las ex-colonias, para continuar explotando sus recursos naturales, así como utilizarlos como mercados para expandir sus economías o como bases para sus ambiciones geopolíticas".

El desarrollo, de esta manera, es una construcción cultural de Occidente que ha colonizado efectivamente "la realidad". En este sentido ha sido fructifero. Sin embargo, los autores del postdesarrollo no están preocupados únicamente del modo cómo el conocimiento es construido, sino además con sus implicaciones materiales en el "mundo real". Con relación a esto, se afirma que el desarrollo ha fracasado. Para Escobar (1995:4), "el discurso y la estrategia del desarrollo han producido su contrario: subdesarrollo masivo y empobrecimiento, opresión y explotación no dadas a conocer, la crisis de la deuda, la hambruna saheliana, incremento de la pobreza, desnutrición y violencia son sólo algunos de los signos más patéticos del fracaso de cuarenta años de desarrollo". Esteva (1987:135) declara agresivamente que "o uno debe ser muy torpe, o muy rico, si no se da cuenta que el desarrollo apesta".

\section{Una evaluación del postdesarrollo}

El enfoque del postdesarrollo indudablemente tiene sus puntos fuertes. Muestra que varias afirmaciones de la universalidad occidental son, de hecho, justificaciones para la dominación de Occidente sobre el sistema mundial. Más aún, correctamente argumenta que la práctica del desarrollo está lejos de ser un proceso neutral y representa fuertes intereses sociales y políticos. Escobar (1995: 10) también ha argumentado convincentemente que el discurso del desarrollo es tan poderoso que las "alternativas al desarrollo" son parte de la misma problemática. Sin embargo, el enfoque del postdesarrollo tiene serias debilidades; a continuación se discutirán sus principales aspectos. 


\section{¿Qué tan novedoso es el argumento del postdesarrollo?}

La utilización que hace el enfoque del postdesarrollo en análisis de discurso es bastante novedosa, llevándolo a afirmar que se constituye en un nuevo punto de vista con relación a las alternativas al desarrollo ${ }^{4}$. Se afirma que, pesar de su radicalidad, la teoría del desarrollo comparte con la teoría de la modernización la creencia en los objetivos del desarrollo, incluso si tienen diferencias de cómo deben lograrse. Los autores del postdesarrollo, en contraste, no comparten la fe que la teoría radical del desarrollo tiene en el papel de un Estado postrevolucionario, ni tampoco perciben el desarrollo (en ninguna forma) como un remedio deseable. La teoría radical del desarrollo intenta presentar estrategias alternativas de desarrollo, mientras que los autores del postdesarrollo afiman que están en la búsqueda de alternativas al desarrollo.

Más adelante se verá claramente que esta distinción es fundamentalmente retórica y sin sentido. Por el momento, un elemento a discutir son las similitudes que el enfoque del postdesarrollo tiene con la teoría de la dependencia ${ }^{5}$. Es en el ámbito de las explicaciones de los motivos que apoyan la construcción del discurso que el enfoque del postdesarrollo retoma las principales ideas de la teoría de la dependencia. Cuando autores como Rahnema argumentan que el desarrollo es el instrumento por medio del cual el Occidente controla sus ex-colonias, están repitiendo lo mismo que varios años antes las teorías del colonialismo interno y de la dependencia afirmaban. Si bien la posición de Escobar es, en varios aspectos, diferente a la indicada, comparte, sin embargo, su punto de vista al afirmar que el Banco Mundial y las empresas multinacionales no son únicamente poderosas, sino además omnipresentes. Como ya es ampliamente conocido, la teoría de la dependencia en su versión más formal ha sido objeto de penetrantes críticas y de nuevas formulaciones (Alfaro, 1990). Sin embargo, el punto más importante con respecto al planteamiento del postdesarrollo es su énfasis en establecer una relación mecánica de dominación entre los países de Occidente y el carácier pasivo adoptado por los países periféricos y la ausencia en su narrativa de un análisis de los diferentes conflictos económicos y políticos que han caracterizado esta relación.

\section{El postdesarrollo y los aspectos metodológicos}

\subsection{La cuestión de la agencia}

El enfoque del postdesarrollo también sufre de inconsistencias metodológicas. En particular su énfasis casi exclusivo en el discurso lo lleva a ambigüedades en su consideración acerca de los agentes del desarrollo, y su antiesencialismo es altamente selectivo. Con relación a lo anterior, como cualquier análisis de discurso existe cierta ambivalencia sobre el problema del poder. En la evaluación de Escobar, "nunca está claro lo que es el poder." El "poder" alterna entre el 
poder en términos de la concepción de Nietzsche, es decir, "el poder inherente en todas las relaciones humanas y las formas específicas de poder como las que se materializan en... instituciones específicas" (Peet 1997:80). Un resultado lógico es un abandono "a la agencia detrás del discurso" (Peet 1997:80). Esta omisión ha sido identificada como una de los puntos débiles del pensamiento de Foucault, ya que constituye un elemento central de su concepción del poder (Best y Kellner 1991:70). Para Foucault —y sus seguidores— el poder no opera en y contra los sujetos individuales; es más es "una máquina en la que todos están atrapados". Así, "el poder viene de abajo; esto es, no existe una oposición binaria que encuadre a dirigentes y dirigidos" (Foucault 1979: 94).

Tal definición de poder es extremadamente problemática. Primeramente, tiene sus implicaciones para toda política de carácter progresista o de otra naturaleza. Si no al mismo tiempo establece la idea de que el poder no tiene sentido. Como lo señala Dews, para que el poder tenga algún sentido, "debe haber algún principio, fuerza o entidad para quienes el poder 'subyugue' o 'domine' y cuya liberación se considere deseable" (Dews 1987: 162).

Escobar busca superar esta omisión tratando de mostrar como la "industria del desarrollo" está compuesta de burócratas que persiguen sus propias metas y objetivos.

El hecho de que las condiciones de la gente no solamente no mejorarán sino que se deterioraran con el tiempo parece no molestar a la mayoría de expertos. En la realidad, estos profesionales han sido colonizados por el discurso del desarrollo, y aquellos que se encontraron insatisfechos con este estado de cosas, tuvieron que luchar por migajas de libertad dentro de éste, con la esperanza de que en el proceso una realidad diferente podría construirse (Escobar 1995: 5).

Con respecto a este tipo de afirmaciones sobre la indiferencia de la "mayoría de los expertos", ninguna evidencia se presenta para respaldarlas. Incluso, estas afirmaciones son muy similares a las desarrolladas por la ideología neoliberal (Pieterse 1998:364), la cual asume que el sector público está compuesto por la suma de individuos con sus propios intereses y que el "bien público" es un mito (Buchanan 1980). Hasta donde las generalizaciones se pueden llevar, las agencias de desarrollo tienden a ser altruistas (pero también paternalistas) en sus intenciones; pero, al mismo tiempo, son, en términos generales ineficientes, al no estar preparadas para enfrentar la naturaleza inesperada y contingente de los resultados del desarrollo. Una cosa es aceptar que las personas involucradas en las agencias y programas de desarrollo son ineficientes, pero otra cosa es la extrema posición de los argumentos Escobar acerca de la práctica de estas personas y la manera como logran sus metas. El resultado es que "en lugar de un análisis de los efectos de los proyectos, los motivos de sus progenitores, individuos e instituciones, son impugnados" (Lehmann 1997: 574). 


\subsubsection{Metodología: el problema del esencialismo}

El enfoque del postdesarrollo es igualmente inconsistente en su antiesencialismo ${ }^{7}$. A pesar que brinda un apoyo total a la diversidad cultural y a la diferencia como fuente de resistencia contra la dominación occidental, el desarrollo mismo es representado en "términos de una hegemonía monolítica" (Peet 1997: 77). Sin consideración de tiempo y espacio, el desarrollo constituye el ejercicio del poder de Occidente sobre los pueblos de "Oriente". Esto tiene dos implicaciones. Primero, sus efectos son similares en cualquier parte del mundo y segundo, que el mundo puede ser dividido entre un Occidente diabólico y un Sur noble. Ambas aseveraciones son problemáticas.

Con respecto al primer punto, si lo aseverado fuera así, entonces, después de cincuenta años de desarrollo, uno podría esperar una convergencia económica, política y cultural en el sistema mundial o al menos un aumento en la homogeneidad del Tercer Mundo. Pero solamente el más ciego de los análisis eurocéntricos podría argumentar que Tanzania, Corea del Sur, la India o El Salvador se han convertido en formaciones similares. Además, los últimos veinte años han visto un incremento en la diversidad del llamado Tercer Mundo; por ejemplo, entre los productores de petróleo y los no productores, el llamado Cuarto Mundo y la emergencia de los "nuevos países industrializados". El surgimiento de los países del Sudeste asiático, probablemente ha sido uno de los fenómenos económicos más importantes de diversidad al interior del mundo periférico a finales del milenio pasado. Sin embargo, este fenómeno es raramente discutido por los autores del postdesarrollo. Paradójicamente, entonces, así como los estudios sobre el desarrollo de orientación marxista se ha vuelto más sensible a la diversidad de la experiencia del desarrollo en los países periféricos (Buttel y McMichael 1994), el enfoque del postdesarrollo trata implícitamente de imponer una nueva relación binaria entre el Primer y el Tercer mundo.

Esta división nos lleva al segundo punto: la convicción de que un Occidente diabólico "envenena" a las sociedades del Tercer Mundo. Dicha relación binaria está presente, por ejemplo, en los trabajos ecofeministas de Mies y Shiva (1993:13), quienes "identifican la libertad con su interacción afectiva con la Madre Tierra". El ideal de estas autoras es de "una perspectiva de subsistencia" basada en la búsqueda de una "sociedad ecológicamente sana, no explotadora, no pratriarcal y autosostenida" (Mies y Shiva 1993: 297), desligada de los demonios del capitalismo occidental y patriarcal. Esta utopía rural está en contraste con la dominación que es intrínseca a todas las formas de desarrollo, lo cual constituye una violencia tanto contra la naturaleza como contra las mujeres que están "todavía incrustadas en la naturaleza" (Shiva 1989: xviii).

La característica sorprendente de estas atrevidas afirmaciones es la manera que ellas presentan un reflejo de la imagen de la llamada posición occidental. La ciencia y la razón son denunciadas como construcciones occidentales contra- 
puestas a los sentimientos e intuiciones superiores que las mujeres poseen. Shiva fracasa al desafiar la idea de que la ciencia y la razón son puras construcciones occidentales. Al hacerlo, no solamente está argumentado que el Iluminismo coincide con los comienzos del colonialismo europeo, sino que el primero dio paso inevitablemente al segundo. Tal idealismo conmovedor, sin embargo, sólo se asume y no existe un intento serio de demostrar esta conexión causal. Por otra parte, otros autores radicales han realizado un análisis más sustancial y crítico de la relación entre el expansionismo europeo y su contrapartida intelectual (Amin 1990). Lo que sí resulta importante mencionar, es que a fines del siglo XIX varios ideólogos europeos se tomaron la tarea de construir "científicamente" narrativas en donde se presentaba un Occidente racional y un Oriente irracional. Tales narrativas eran, en consecuencia, claramente justificadoras del expansionismo europeo (Said 1987).

\subsubsection{Postdesarrollo y política}

Los comentarios realizados sobre el esencialismo tienen serias implicaciones para las opciones políticas defendidas por el postdesarrollo. En este apartado se hará énfasis en cuatro puntos íntimamente relacionados. Los problemas acerca de la tecnología, el relativismo, el entusiasmo sobre lo local y el silencio sobre los movimientos sociales son puntos discutidos en la medida en que se constituyen en componentes claves de la retórica del postdesarrollo.

\subsubsection{La tecnología}

Los autores del postdesarrollo tienden a emplear una posición extremadamente antitecnológica (Ullrich, 1992) y en tal sentido, ignoran las posibilidades que la tecnología puede brindar. Ciertamente, las nuevas tecnologías pueden generar efectos contradictorios. Uno de ellos sería, por un lado, la forma como reduce el tiempo de trabajo necesario e incrementa el consumo, mientras por el otro, simultáneamente descansa en la explotación del trabajo y del medio ambiente. Una cosa es reconocer los sesgos sociales de la tecnología, pero otra es llegar a concluir lo que las sociedades harían sin ella.

El enfoque del postdesarrollo sobre la tecnología tiende a mezclar dos posiciones, una posición antitecnológica y otra en contra de la tecnología foránea. En cualquier caso, ellos argumentan que el desarrollo tecnológico que podría ser apoyado es el auténticamente local. Pero esta posición (lo local es bueno y lo foráneo malo, respecto a la determinación tecnológica) hace abstracción de las motivaciones que existen en la introducción de tecnologías nuevas y los efectos que estas tienen sobre diferentes grupos (clases, géneros, etc.) sin importar su origen. Por supuesto, la importación de tecnología puede ser, en muchos casos, inapropiada, pero la razón por la cual ciertos grupos poderosos deciden su importación nada tenga que ver con que lo "local sea bueno" y todo esté relaciona- 
do con aumentar sus ganancias. Además, puede haber casos en los que, independientemente de la lógica que la respalda, la importación de tecnología o bienes determinados de los países occidentales tenga efectos beneficiosos. Uno podría preguntarse cuál sería el punto de vista de estos intelectuales si alguna compañía farmacéutica produce en los próximos años un medicamento que cure el virus del SIDA. Al puntualizar algunos ejemplos de lo útil que puede resultar la importación de tecnología extranjera, no significa que todas las importaciones sean buenas, pero tampoco los efectos producidos por los ejemplos negativos significan que todas las importaciones son malas. El punto es que el problema de la importación de tecnología y de bienes manufacturados es una cuestión abierta. Sin embargo, para los agrupados en el postdesarrollo este es un asunto cerrado.

Además, dejando a un lado el debate de lo local y lo foráneo con relación a la tecnología, todavía se mantiene un sesgo antitecnológico entre los autores mencionados (Álvarez, 1992). Esta posición es tan tecnológicamente determinista como la fuerte posición desarrollista asumida por la teoría de la modernización en los Estados Unidos durante los cincuenta del siglo recién pasado, ya que, mientras los primeros aseguran que la tecnología moderna genera un sentimiento de pérdida, la última la muestra como un signo de progreso. Ambas posiciones, sin embargo, comparten el punto de vista que la tecnología determina el resto de los procesos sociales.

Atribuir todos los males del Tercer Mundo al discurso del desarrollo también resulta muy problemático. Así, por ejemplo, cuando Escobar (1995:4) se muestra preocupado con el problema de la deuda, la pobreza, la hambruna, la desnutrición y la violencia uno se pregunta si está utilizando un criterio muy particular o está utilizando un enfoque elaborado al interior del discurso del desarrollo. Discutir dicho problema nos lleva a plantear uno de los más importantes componentes del postdesarrollo: el relativismo cultural.

\subsubsection{El relativismo}

Estos problemas también son componentes ambiguos en los trabajos de Foucault. En ellos existe una tensión no resuelta entre sus afirmaciones acerca de la inseparabilidad del conocimiento y el poder y su propia posición política. Foucault argumenta, por un lado, que el poder es inseparable del conocimiento y las verdades que se defienden, mientras por el otro, argumenta que este debe ser resistido. Sin embargo, cono Fraser lo señala, el pensador francés adopta un concepto de poder que le permite la condenación teórica de toda característica cuestionable de las sociedades modemas. Pero, al mismo tiempo, y por otro lado, su retórica traiciona la convicción que las sociedades modernas no poseen ninguna característica que las redima. Claramente, lo que Foucault necesita $-y$ lo necesita desesperadamente-, son criterios normativos para distinguir formas aceptables de poder de las que son inaceptables (Fraser 1989:33). 
Siguiendo esta aguda observación, lo que Foucault y sus seguidores necesitarían para justificar su propia posición política es una teoría que rompa con la relación inseparable entre poder y conocimiento, y de esa forma demostrar cómo algunas teorías son apoyadas por falsedades, mientras otras, con más asideros en la verdad, se encuentran al lado de la resistencia (Dews 1987: 189-91). En otras palabras, una política de resistencia del tipo de la defendida por Foucault descansa en última instancia en la distinción entre verdad e ideología. Este punto ha sido retomado por feministas que no son seguidoras incondicionales de Foucault, quienes, en tanto simpatizantes de un falso historicismo de "verdades" (sexistas), también puntualizan que un histcricismo total socava las bases de una política feminista (Soper 1993:45).

Las mencionadas tensiones penetran los textos de los autores del postdesarrollo. Consistentes con su crítica del lluminismo como fuente de la dominación europea, Esteva y Prakash (1997:282) argumentan que cualquier pretensión "universalista" de los derechos humanos, es en realidad propia del mundo occidental. Dicho universalismo es así una pretensión de poder, una manera por medio de la cual el Occidente ejerce su dominación en el sistema mundial. En su lugar, estos autores recomiendan una estrategia de resistencia local (y emplean el estandarte de "piensa en local, actúa en lo local"), "una estrategia activa que se oponga a todos los abusos de poder, tanto en sus formas pre-modernas como las modemas, en todas sus manifestaciones" (Esteva y Prakash 1997:284). En el mismo sentido, se argumenta que dicha posición es totalmente compatible con una oposición a los derechos humanos universales, pero una oposición a "todos los abusos de poder" como la defendida por estos parece notablemente cerca a una posición universalista.

Por supuesto, al puntualizar las implicaciones políticas a las que se puede arribar con los argumentos expresados no significa que el desarrollo sea en parte una construcción discursiva. Sin embargo, el enfoque del postdesarrollo no enfrenta este problema de manera convincente. El hecho de admitir que los "objetos" o "sujetos" del desarrollo sólo pueden ser conocidos por medio del discurso, no es lo mismo que pretender (como los autores del postdesarrollo algunas veces lo sostienen) que también pueden ser reducidos a discurso.

Así, mientras el giro discursivo en los estudios del desarrollo $\longrightarrow$ de los cuales el enfoque del postdesarrollo es un componente- es, en muchos sentidos, bienvenido, pero no a expensas del análisis materialista. Si bien es cierto que las ideas son producidas históricamente, "no se puede concluir... que no podamos saber que lo conocido (bajo descripciones particulares) existe y actúa independientemente de estas descripciones" (Bhaskar 1989:152). Mientras que el conocimiento de la realidad depende de una teoría, la realidad misma no está supeditada a ninguna teoría. Así, la historización del conocimiento no involucra un juicio relativista en el cual todo es reducido a discurso". 


\subsubsection{Los movimientos sociales y la política sin compromiso}

La oposición binaria entre lo global/demonio y lo local/noble es llevada hasta sus últimas consecuencias en la evaluación de los movimientos sociales en el Tercer Mundo. Si bien Escobar muestra convincentemente cómo incluso el pensamiento más radical del desarrollo no se encuentra fuera del discurso del desarrollo, pero más adelante, sin mayor elaboración, afirma que de alguna manera los movimientos sociales pueden lograr dicha autonomía (Escobar 1995: 222-3). Sin embargo, no existe una disposición de evaluar la política específica de estos movimientos, ya que el efecto de tal posición podría ser "mantenerse dentro del mismo modelo de pensamiento que produjo el desarrollo y lo mantiene en su lugar" (Ibid.: 222). Consciente de este peligro, Slater (1994:29) argumenta que "tal vez, después de todo, todo lo que se puede decir es que los movimientos están allí, y que se mantienen en movimiento". Pero esta posición, de nuevo, muestra el inconsistente antiesencialismo del enfoque en discusión.

En los análisis del grupo de intelectuales vinculados a la noción del postdesarrollo, se encuentra su simpatía hacia los movimientos identificados con la tradición o su apoyo a una nueva apertura a la política. Esta última posición, sin embargo, es tan vaga y aperturista que libra de cualquier compromiso político a quienes la defienden. Cuando Rahnema (1997) argumenta que el fin del desarrollo "representa un llamado a las 'personas buenas' de cualquier parte a pensar y trabajar juntos", nos deja la sensación de una política banal y vacía en la mejor tradición de la canción We are the World que fuera tan popular a mediados de los años ochenta. En lugar de una posición política que se empeñe en criticar las desigualdades materiales, lo que tenemos es una era de postdesarrollo donde "las personas deberían ser simpáticas y amables en sus relaciones". Una vez más, dicha posición cómoda puede ser vinculada a una perspectiva en la cual todo puede ser reducido fácilmente al discurso. Así, algunos enfoques sobre la pobreza, una construcción del discurso del desarrollo, "trata... a las personas como objetos abstractos, datos estadísticos que pueden moverse de abajo hacia arriba en los cuadros del 'progreso'"(Escobar 1995: 44). Esto puede llevar a un tratamiento tecnocrático de la pobreza en donde la gente "abandonada" por el desarrollo sería el objetivo, con la esperanza de que el "efecto cascada" eventualmente tendrá lugar. Pero el problema con la mayoría de estos autores es su insinuación de que este es el único enfoque sobre el fenómeno de la pobreza (mientras, simultáneamente, utilizan estadísticas oficiales para intentar demostrar el fracaso del desarrollo). Por otra parte, en una visión mucho más amplia, la mayoría de los enfoques radicales e históricos del desarrollo enfatizan en las relaciones de producción y distribución para demostrar las relaciones materiales que existen entre riqueza y desigualdad y la vinculación de éstas a la dinámica de la economía capitalista mundial. 


\section{Conclusión: La unidad contradictoria del desarrollo}

El enfoque del postdesarrollo se caracteriza por ser incompleto en una variedad de aspectos. En sus versiones más extremas refleja, a pesar de su evidente preocupación y sensibilidad, un orientalismo invertido. En su versión más matizada - Escobar - muestra el poder del discurso del desarrollo, pero, al misino tiempo, su texto evidencia serias inconsistencias. Así, algunas veces los autores asociados a este enfoque, argumentan que los indicadores del desarrollo oficiales son productos sesgados del discurso del desarrollo y, por lo tanto, los rechazan; pero, al mismo tiempo, estos indicadores son utilizados para expresar su punto de vista de que el desarrollo ha fracasado. Incluso entonces, son utilizados para hacer afirmaciones muy generales basadas en una falta de rigor metodológico. Los que nos brinda tal ejercicio intelectual es, ya sea una posición donde todo es reducido a discurso $y$, por lo tanto, un relativismo total, o un empirismo que no resiste un escrutinio práctico seric. De igual manera, si bien demuestra fehacientemente la naturaleza perversa del discurso del desarrollo, Escobar realiza un giro rápido y en forma poco convincente afirma que los movimientos sociales se encuentran de alguna manera fuera del desarrollo.

El enfoque del postdesarrollo también tiende a lavarse las manos con respecto a la lógica política de estos movimientos, afirmando que todos las pretensiones de conocimiento son igualmente pretensiones de poder. Pero este punto de vista "fracasa, al no distinguir entre las totalidades legítimas e ilegítimas y macroteorias; por ejemplo, entre modos abiertos y heterogéneos de análisis que sitúan particularidades discretas dentro de un contexto común de determinación, y modos homogéneos que destruyen las diferencias entre fenómenos diversos" (Best y Kellner 1991:72). De acuerdo con estos criterios, la teoría de la modernización puede considerarse una totalidad ilegítima, pero no puede decirse lo mismo acerca de los enfoques críticos o radicales al interior del pensamiento del desarrollo.

Las bases para un enfoque alternativo del desarrollo podrían perfectamente utilizar la noción de "hibridización" presente en las contribuciones actuales de varios académicos latinoamericanos (García Canclini, 1990; Quijano, 1988; Ianni, 1996) y en las contribuciones cotidianas de amplios sectores de las clases populares y de la sociedad civil en general. De lo que se trata es de rescatar los principales aportes intelectuales que permitan a las sociedades del Tercer Mundo forjar sus propias lógicas económicas y sociales en un contexto de justicia social y de participación democrática real. La búsqueda de opciones viables y concretas no descansa en posiciones contemplativas o románticas cuyo referente sea la moda intelectual, los autores del postdesarrollo lamentablemente caen en las redes del "monstruo" que intentan deconstruir, al forzar la interpretación de realidades sumamente complejas para que éstas respondan a perspectivas cognoscitivas de inspiración postestructuralista, cada vez más agotadas en el ámbito académico del Norte. 
La noción del postdesarrollo, en definitiva, constituye un componente de la historia al interior del discurso del desarrollo. Las diferentes ideas acerca del desarrollo, por lo tanto, necesitan ser evaluadas no en términos de su supuesta autonomía respecto al discurso del desarrollo, sino en términos de sus propuestas viables y concretas para mejorar las condiciones y la calidad de vida de la mayor parte de las clases populares del planeta.

\section{Notas}

1. Algunos autores (Booth 1992) han argumentado que existe un vínculo muy estrecho entre el impasse en la teoría y la crisis del desarrollo en la vida real. Estos dos no son, ni complementamente inseparables ni tampoco completamente similares. El argumento de que una "correcta" teoría del desarrollo automáticamente asegura una política "correcta" ignora la cuestión del poder y la agencia en una situación concreta. Existen importantes diferencias entre teoría y modelo de desarrollo. Los autores del postdesarrollo fracasan al no analizar esta distinción adecuadamente.

2. Al hacer referencia al enfoque del postdesarrollo en singular, se corre el riesgo de caricaturizar las ideas de un grupo importante de autores. Esto es incluso más notable debido al compromiso manifiesto de este enfoque con respecto a la diversidad y el pluralismo. Sin embargo, nos parece legítimo asociarlos, ya que la mayoria de ellos comparten las ideas fundamentales y por lo tanto se puede utilizar el singular.

3. Dicho de una forma más precisa, la noción del desarrollo como la conciben los teóricos del postdesarrollo. Por otra parte, quedará claro más adelante que la noción del desarrollo se localiza mucho antes del período posterior a la Segunda Guerra Mundial.

4. Ver, por ejemplo, los comentarios de Escobar (1995: 99-100) acerca del trabajo de uno de los más prominentes teóricos marxistas del desarrollo, Samir Amin.

5. En esta discusión se utiliza el concepto de teoría de la dependencia de manera muy sintética. Vale la pena mencionar que este paradigma posee múltiples versiones.

6. Esta posición neoliberal nos lleva al punto de vista que la participación de los Estados debe minimizarse y que a las fuerzas del mercado [capitalista] se les permita florecer y expandirse. En tal sentido, los programas de ajuste estructural son justificados por este planteamiento. Sí bien el enfoque del postdesarrollo es crítico de estos programas, su crítica al desarrollo es, algunas veces, sorprendentemente similar a la crítica neoliberal.

7. El esencialismo es a menudo percibido como una de las grandes debilidades de las teorías de la modernidad. Los postestructuralistas que hacen tal afirmación, son, con frecuencia, "culpables" de su propia crítica. El punto en discusión en el presente artículo acerca del esencialismo es mostrar las inconsistencias de los autores del postdesarrollo y no un ataque al esencialismo per se.

8. Los decontruccionistas radicales - muy comunes en los Estudios Culturales - han utilizado una "perspectiva radical" para hacer la ridícula afirmación de que la investigación empírica no es necesaria. Dicha posición —la separación radical entre hechos y teoría- es realmente una "imagen-reflejo" de la posición positivista-empirista.

\section{Referencias Bibliográficas}

Alfaro, S. O., Theories of Development and Social Change. Tesis de Maestría en Sociología, Universidad de Regina, Canadá, 1990. 
Álvarez, C., Science, Development and Violence: The Revolt Against Modernity, Delhi: Oxford University Press, 1992.

Amin, S. Eurocentrism, New York, Monthly Review, 1990.

Best, S. y D. Kellner, Postmodern Theory, London, Macmillan, 1991.

Bhaskar, K., Reclaiming Reality, London, Verso, 1989.

Booth, D., Social Development Research: An Agenda for the 1990's, European Journal of Development Research, Vol. 4, No. 1, 1-35.

(ed.) Rethinking Social Development, London: Longman, 1994.

Buchanan, J., Rent-seeking and Profit-seeking, en J. Buchanan, R. Tollison y G. Tullo (eds.), Towards a Theory of the rent-seeking Society, Texas: A \& M University Press, $1-21,1980$.

Buttel, F. y P. McMichael, Reconsidering the Explanandum and Scope of Development Studies: Towards a Comparative Sociology of State-Economy Relations, en D. Booth (ed.) Rethinking Social Development, London, Longman, 42-61, 1994.

Chenery, H. et. al., Redistribution with Growth, Oxford, Oxford University Press, 1974.

Dews, P., Logic of Desintegration, London, Verso, 1987.

Escobar, A., Encountering Development, Princenton, NJ, Princenton University Press. 1995.

Esteva, G., Regenerating People's Space en Alternatives, Vol. 10, No. 3, 125-52, 1987.

Esteva, G. y M.S. Prakash, From Global Thinking to Local Thinking, en Rahnema y Bawtree (eds.), 1997, 277-89.

Foucault, M., Discipline and Punishment, London, Tavistock, 1977. History of Sexuality, Vol. 1, Harmondsworth: Penguin, 1979. Power/Knowledge, Brighton, Harvester, 1980.

Fraser, N., Unruly Practices: Power, Discourse and Gender in Contemporary Social Theory, Cambridge, Polity, 1989.

García Canclini, N., Culturas hibridas: Estrategias para entrar y salir de la modernidad, México, Grijalbo, 1990.

Ianni, O., Teorías de la Globalización, México, Siglo XXI, 1996.

Kiely, R., Sociology and Development: The Impasse and Beyond, London, UCI Press, 1995.

Lal, D., The Poverty of Development Economics, London, Institutc of Economic Affairs, 1984.

Lehmann, D., An Opportunity Lost: Escobar's Deconstruction of Development, The .Journal of Development Studies, Vol. 33, No.4, 568-78.

Mies, M. y V. Shiva, Ecofeminism, London: Zed, 1993.

Peet, R., Social Theory, Postmodernism and the Critique of Development, en G. Benko y U. Strohmayer (eds.), Space and Social Theory, Oxford, Blackwell, 1997, 343-73.

Pieterse, J.N., My Paradigm or Yours? Alternative Development, Post-development, Reflexive Development, Development and Change, Vol. 29, 343-73.

Prebish, R. Comercial Policy in the Underdeveloped Countries, en American Economic Review, Vol. 44, 251-73.

Quijano, A., Modernidad, identidad y utopia en América Latina, Socicdad y Política edic., Lima, 1988.

Rahnema, M. y V. Bawtree (eds.), The Post-Development Reader, London, Zcd, 1997.

Sachs, W., Introduction, en Sachs (ed.) The Development Dictionary, London, Zcd, 1992. 
Said, E., Orientalism, New York, Vintage, 1987.

Slater, D., Power and Social Movements in the Other Occident, en Latin American Perspectives, Vol. 21, No. 2, 1994, 11-37.

Soper, K., Productive Contradictions en C. Ramazanoglu, Up Against Foucault, London, Routledge, 1993, 29-50.

Ullrich, O., Technology en Sachs (ed.) 1992, 275-87.

Digitalizado por Biblioteca "P. Florentino Idoate, S.J." Universidad Centroamericana José Simeón Cañas 